\title{
Plasmonic Skyrmion Lattice Based on the Magnetoelectric Effect
}

\author{
X.-G. Wang, ${ }^{1}$ L. Chotorlishviliø ${ }^{2}$ N. Arnold $\odot{ }^{3}$ V. K. Dugaev, ${ }^{4}$ I. Maznichenko, ${ }^{2}$ J. Barnas,,${ }^{5,6}$ \\ P. A. Buczek, ${ }^{7}$ S. S. P. Parkin, ${ }^{8}$ and A. Ernst $\oplus^{8,9}$ \\ ${ }^{1}$ School of Physics and Electronics, Central South University, Changsha 410083, China \\ ${ }^{2}$ Institut für Physik, Martin-Luther Universität Halle-Wittenberg, D-06120 Halle/Saale, Germany \\ ${ }^{3}$ Soft Materials Lab, Linz, Institute of Technology LIT, Johannes Kepler University Linz, Altenberger Straße 69, 4040 Linz, Austria \\ ${ }^{4}$ Department of Physics and Medical Engineering, Rzeszów University of Technology, 35-959 Rzeszów, Poland \\ ${ }^{5}$ Faculty of Physics, Adam Mickiewicz University, ul. Uniwersytetu Poznańskiego 2, 61-614 Poznań, Poland \\ ${ }^{6}$ Institute of Molecular Physics, Polish Academy of Sciences, ul. M. Smoluchowskiego 17, 60-179 Poznańn, Poland \\ ${ }^{7}$ Department of Engineering and Computer Sciences, Hamburg University of Applied Sciences, \\ Berliner Tor 7, 20099 Hamburg, Germany \\ ${ }^{8}$ Max Planck Institute of Microstructure Physics, Weinberg 2, D-06120 Halle, Germany \\ ${ }^{9}$ Institute for Theoretical Physics, Johannes Kepler University, Altenberger Straße 69, 4040 Linz, Austria
}

(Received 29 May 2020; revised 4 August 2020; accepted 1 October 2020; published 23 November 2020)

\begin{abstract}
The physical mechanism of the plasmonic skyrmion lattice formation in a magnetic layer deposited on a metallic substrate is studied theoretically. The optical lattice is the essence of the standing interference pattern of the surface plasmon polaritons created through coherent or incoherent laser sources. The nodal points of the interference pattern play the role of lattice sites where skyrmions are confined. The confinement appears as a result of the magnetoelectric effect and the electric field associated with the plasmon waves. The proposed model is applicable to yttrium iron garnet and single-phase multiferroics and combines plasmonics and skyrmionics.
\end{abstract}

DOI: 10.1103/PhysRevLett.125.227201

Surface plasmon polaritons (SPPs) are surface electromagnetic waves that exist at the interface between two materials with opposite signs of the dielectric function $\epsilon(\omega)$. SPPs propagate along the surface and exponentially decay in both media away from the interface. The plane waves are $\boldsymbol{p}$ polarized, i.e., the electric field $\boldsymbol{E}$ has only $x$ (along the propagation direction) and $z$ (normal to the surface) components, and the $z$ component is typically dominant $[1,2]$. One can manipulate the SPPs within the plane using their reflections from the edges or grooves in a metal film or by modulation of the film thickness or substrate and superstrate properties [3-7]. As mentioned above, SPPs inherently possess a dominant normal $z$ component of the electric field, which is an important property not easily achieved by other means. In addition, the wavelength of an SPP is always shorter than that in the vacuum, which allows one to attain subwavelength precision. SPPs are relatively well-studied with the existing experimental tools for their generation and manipulation. As strongly confined surface waves, they can be used in

Published by the American Physical Society under the terms of the Creative Commons Attribution 4.0 International license. Further distribution of this work must maintain attribution to the author(s) and the published article's title, journal citation, and DOI. Open access publication funded by the Max Planck Society. compact micro- and optoelectronic devices. Interestingly, SPPs were used recently to create electric-field skyrmions [8]. The interference of two contrapropagating plasmons creates a standing-wave plasmon with similar properties, including the dominant $z$ component of the electric field. Four appropriately arranged waves result in a lattice-type pattern (see Fig. 1). The smallest half-width of the maxima, i.e., of the plasmonic lattice sites, can be of the order of $\lambda_{x} / 4$ (depending on the definition), which can reach 70$100 \mathrm{~nm}$, and the distance between them (i.e., interference period) is about $\lambda_{x} / 2$ (see [7]). In this Letter, we propose to use such plasmonic lattices in order to construct a new type of matter: the plasmonic skyrmion lattice. We show that magnetic skyrmions can be trapped by the electric field of the surface plasmon polaritons to form the plasmonic skyrmion lattice. By analogy with cold atoms and optical lattices, we propose the model of the plasmonic skyrmion lattice based on the magnetoelectric effect. Because of this effect, the electric field of an SPP couples to the ferroelectric polarization. The latter depends on the electric field, so the effect is quadratic. The characteristic oscillation frequency of the SPP mode is of the order of $100 \mathrm{THz}$, while the magnetic system does not react to frequencies higher than $1 \mathrm{THz}$. However, the skyrmion does not stay in its energy minimum but is trapped in the region near the largest spatial variations of the electric field. Temporal field oscillations lead to a fast, almost unnoticeable small-amplitude 


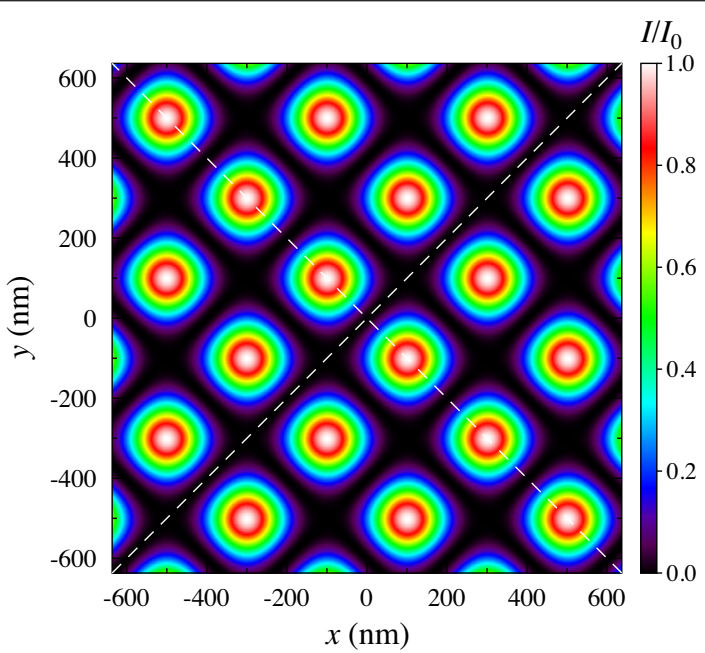

FIG. 1. The plasmonic confinement lattice. The plot shows the distribution of the plasmonic wave intensity $I=2 E_{l s}^{2}\left[\cos \left(k_{x} x+\right.\right.$ $\left.\left.\varphi_{x}\right)+\cos \left(k_{y} y+\varphi_{y}\right)\right]^{2}$ normalized to its maximal value $8 E_{l s}^{2}$ in the center of a given lattice site. The radius of the lattice sites is about 70-100 nm, and the distance between them 250-300 nm. The distance between lattice sites exceeds the characteristic skyrmion interaction length (see below). Therefore skyrmions confined in the lattice sites can be treated as independent objects. Nevertheless, to explore the robustness of the confinement, we also consider the case when the distance between lattice sites is comparable to the skyrmion interaction length.

alternating winding or unwinding optical-frequency trembling of the skyrmion around the points of maximum intensity. Therefore, confinement depends on the spatially inhomogeneous intensity of the time-averaged SPP field. Similar optical lattice can be created using higher-order optical Gaussian modes (see Supplemental Material [9] for more details). Confined skyrmions are expected to play the role of fundamental elements of memory storage devices and high functional quantum information protocols. Skyrmionics is a relatively new topic that emerged in the last decade, and it draws inspiration from the fundamental field theory studies of 1960s and 1970s [29-31]. Belavin and Polyakov [32] were the first to study the Heisenberg field functional and observe that the antisymmetric exchange, i.e., the Dzyaloshinskii-Moriya interaction, minimizes the energy. Various interesting aspects of skyrmionics have been already studied in the recent literature [33-48]. We note recent interest in the biskyrmion states embedded in the surface of a topological insulator [49] and chimney ladder ferromagnet [50]. The advantage of our model is that it is not limited to two skyrmion states and that the plasmonic skyrmion lattice is stable independently of the attractive or repulsive character of interaction between the skyrmions. The theory we present below is experimentally feasible for single phase multiferroics, i.e., $\mathrm{Cu}_{2} \mathrm{OSeO}_{3}$ (see [35]) and yttrium iron garnet, materials that also show multiferroicity $[51,52]$. The distance between plasmonic lattice sites is about $300 \mathrm{~nm}$ and exceeds the skyrmion-skyrmion interaction length. Thus,
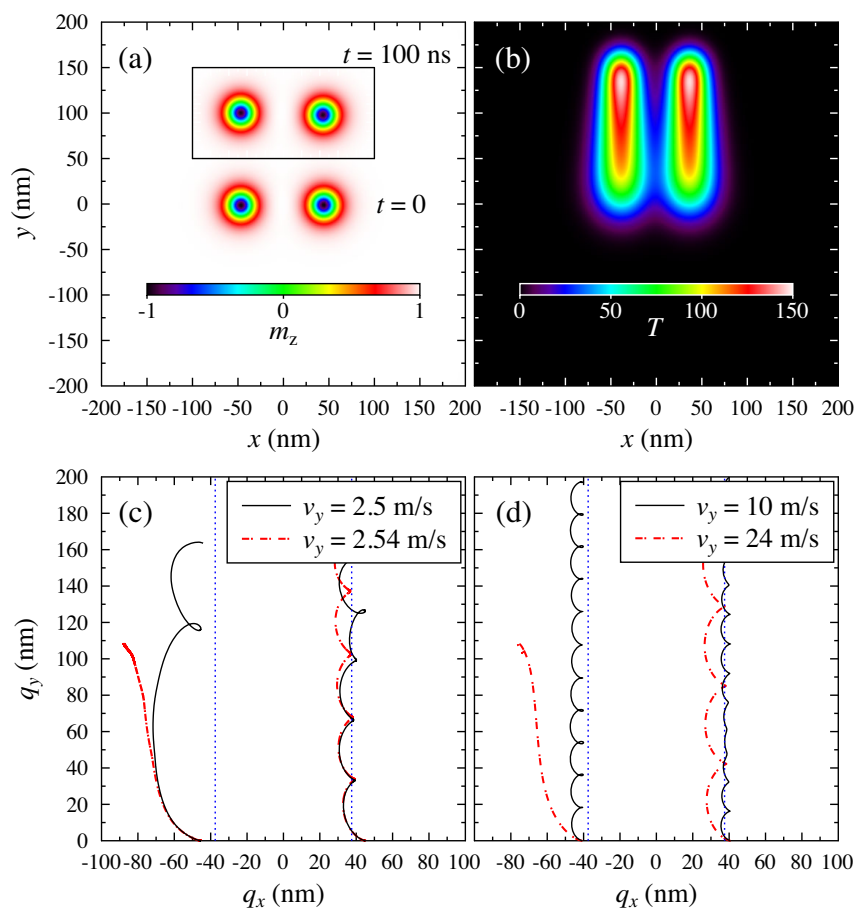

FIG. 2. (a) The center of the plasmonic beam $\left(x_{0}, y_{0}\right)$ is steered with the velocity $v_{y}=1 \mathrm{~m} / \mathrm{s}$ and $E_{0}=0.2 \mathrm{MV} / \mathrm{cm}$. The spatial profile of $m_{z}$ at time $t=0$ and $t=100 \mathrm{~ns}$ (from micromagnetic simulations). The color specifies the value of the $m_{z}$ component of magnetization. (b) The temperature profile $T(x, y, t)$ induced by plasmonic surface waves. The maximum of the temperature coincides with interference peaks of plasmonic surface waves (plasmonic lattice sites). The drift of the interference peaks with the velocity $v_{x}=8 \mathrm{~m} / \mathrm{s}$ forms the temperature pattern. The maximal temperature for the electric field $E_{0}=0.8 \mathrm{MV} / \mathrm{cm}$ and $l_{g}=37.5 \mathrm{~nm}$ is $T_{m}=150 \mathrm{~K}$. (c) Centers of the plasmonic beam $\left(x_{0}, y_{0}\right)$ are steered (blue dashed lines) along the $y$ axis. The curly trajectories of two skyrmion centers (from micromagnetic simulations) are tracked for different velocities of the plasmonic beam: $v_{y}=2.5 \mathrm{~m} / \mathrm{s}$ (black solid lines) and $2.54 \mathrm{~m} / \mathrm{s}$ (red dashed lines). Blue lines represent the trajectory of the two laser peak centers. Here, we use $E_{0}=0.2 \mathrm{MV} / \mathrm{cm}$ and $l_{g}=37.5 \mathrm{~nm}$. (d) By solving Thiele equations (see Supplemental Material [9]), the trajectories of two skyrmion centers are tracked for different velocities of the plasmonic beam: $v_{y}=10 \mathrm{~m} / \mathrm{s}$ (black solid lines) and $24 \mathrm{~m} / \mathrm{s}$ (red dashed lines) for $E_{0}=0.8 \mathrm{MV} / \mathrm{cm}$ and $l_{g}=37.5 \mathrm{~nm}$.

the skyrmions confined in the plasmonic lattice (see Fig. 1) are independent. However, to study the robustness of the confinement, we consider dynamical effects, i.e., the steering of two lattice sites with confined skyrmions at a distance smaller than the interaction length [Fig. 2(a)]. The free energy of the system has the form

$$
F[\mathbf{m}]=\int\left[A_{\mathrm{ex}}(\boldsymbol{\nabla} \mathbf{m})^{2}-\mu_{0} M_{s} m_{z} H_{z}+E_{\mathrm{me}}\right] d \mathbf{r} .
$$

Here, the first, second, and third terms describe exchange, Zeeman, and magnetoelectric interactions, respectively. 
Because of the magnetoelectric (ME) coupling, $E_{\mathrm{me}}=-\mathbf{E} \cdot \mathbf{P}$, the applied external electric field $\mathbf{E}$ coupled to the ferroelectric polarization $\mathbf{P}=c_{E}[(\mathbf{m} \cdot \nabla) \mathbf{m}-\mathbf{m}(\nabla \cdot \mathbf{m})]$ (with the ME coupling parameter $c_{E}$ ) plays the role of an effective Dzyaloshinskii-Moriya (DM) constant [53,54], $D=c_{E}|\mathbf{E}|$. The above formula for $\mathbf{P}$ is valid for a small magnetization gradient, low temperatures, and in the linear approximation (small $P$ and $E$ ); otherwise, nonlinear terms in the free energy may play a significant role. In turn, the skyrmion dynamics is governed by the stochastic Landau-Lifshitz-Gilbert (LLG) equation

$$
\frac{\partial \mathbf{m}}{\partial t}=-\gamma \mathbf{m} \times\left(\mathbf{H}_{\mathrm{eff}}+\mathbf{h}_{l}\right)+\alpha \mathbf{m} \times \frac{\partial \mathbf{m}}{\partial t},
$$

where $\gamma$ is the gyromagnetic ratio and $\alpha$ is the phenomenological Gilbert damping constant. The effective field $\mathbf{H}_{\text {eff }}$ consists of the exchange field, the DM field, and the applied external magnetic field $\mathbf{H}_{\text {eff }}=\left(2 A_{\text {ex }} / \mu_{0} M_{s}\right) \nabla^{2} \mathbf{m}-$ $\left(1 / \mu_{0} M_{s}\right)\left(\delta E_{\mathrm{me}} / \delta \mathbf{m}\right)+H_{z} \mathbf{z}$. The temperature in the LLG equation is introduced through the correlation function of the thermal random magnetic field $\mathbf{h}_{l},\left\langle h_{l, p}(t, \mathbf{r}) h_{l, q}\left(t^{\prime}, \mathbf{r}^{\prime}\right)\right\rangle=$ $\left(2 k_{B} T \alpha / \gamma \mu_{0} M_{s} V\right) \delta_{\mathrm{pq}} \delta\left(\mathbf{r}-\mathbf{r}^{\prime}\right) \delta\left(t-t^{\prime}\right)$, where $p, q=x, y$, $z, k_{B}$ is the Boltzmann constant, and $V$ is the volume of the sample. The total electric field $\mathbf{E}=\mathbf{E}_{0}+\mathbf{e}_{z} E_{l s}(x, y, z)$ consists of two terms. The constant external field $\mathbf{E}_{0}=$ $\left(0,0, E_{z 0}\right)$ coupled to the ferroelectric polarization is the essence of DM constant, $D_{0}=c_{E} E_{z 0}$, and stabilizes the skyrmion magnetic texture. The nonuniform plasmonic field $\mathbf{e}_{z} E_{l s}(x, y, z)\left(\mathbf{e}_{z}\right.$ is the unit polarization vector of electric field) contributes to the inhomogeneous electric torque (IET) in Eq. (2): $-\gamma \mathbf{m} \times\left\{-\left[\delta E_{\mathrm{me}}\left(\partial_{r} E_{z}\right)\right] /\left[\mu_{0} M_{s} \delta \mathbf{m}\right]\right\}=$ $-\left(\gamma c_{E} \partial_{r} E_{z} / \mu_{0} M_{s}\right) \mathbf{m} \times\left(\mathbf{m} \times \mathbf{p}_{\mathbf{E}}\right)$. The vector $\mathbf{p}_{\mathbf{E}}=\mathbf{e}_{r} \times \mathbf{e}_{z}$ is set by $\mathbf{e}_{z}$, and $E_{z}$ is spatially varying along $\mathbf{e}_{r}$, where $\mathbf{e}_{r}=\left(\mathbf{e}_{x}+\mathbf{e}_{y} / \sqrt{x^{2}+y^{2}}\right)$ is the unit vector. Note that the $z$ component of the nonuniform field is dominated by the plasmonic polariton field. The expression of IET is identical to the standard spin transfer torque $-c_{j} \mathbf{m} \times(\mathbf{m} \times \mathbf{p})$, with $c_{j}=\left(\gamma c_{E} \partial_{r} E_{z} / \mu_{0} M_{s}\right)$. For more details concerning the IET, we refer to [55]. The central role in the formation of a bounded skyrmion lattice is played by the IET and the plasmon field. In numerical calculations, we assume the following set of parameters: the saturation magnetization $M_{s}=1.4 \times 10^{5} \mathrm{~A} / \mathrm{m}$, the exchange constant $A_{\mathrm{ex}}=3 \times$ $10^{-12} \mathrm{~J} / \mathrm{m}$, the ME coupling strength $c_{E}=0.9 \mathrm{pC} / \mathrm{m}$, and the Gilbert damping constant $\alpha=0.001$. The Néel-type skyrmion is stabilized by the electric and magnetic fields, $E_{z 0}=2.5 \mathrm{MV} / \mathrm{cm}$ and $H_{z 0}=1 \times 10^{5} \mathrm{~A} / \mathrm{m}$. Two plasmonic lattice sites in question are described through the twomaximum Gaussian beam $E_{l s}(x, y)=E_{0}\left(\exp \left\{-\left[\left(x-l_{g}-\right.\right.\right.\right.$ $\left.\left.\left.x_{0}\right)^{2}+\left(y-y_{0}\right)^{2}\right] /\left[\sigma_{0}^{2}\right]\right\}+\exp \left\{-\left[\left(x+l_{g}-x_{0}\right)^{2}+\left(y-y_{0}\right)^{2}\right] /\right.$ $\left.\left.\left[\sigma_{0}^{2}\right]\right\}\right)$. Here, $\left(x_{0}, y_{0}\right)$ is the center of two plasmonic lattice sites and $2 l_{g}$ is the distance between the two sites.
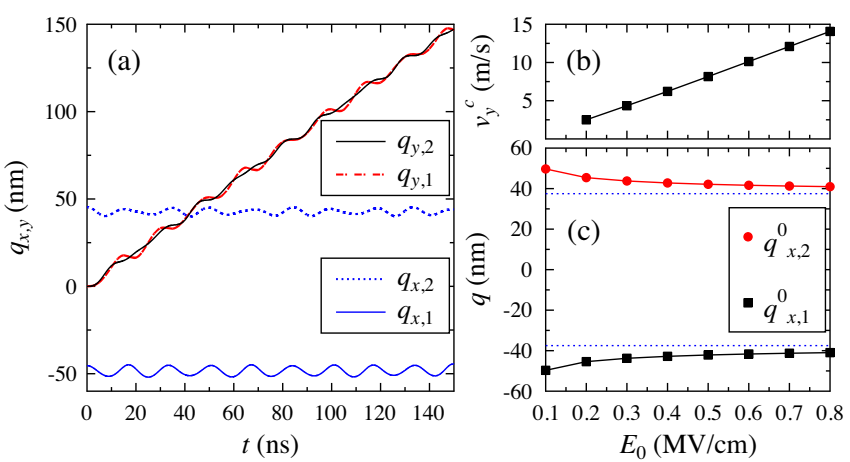

FIG. 3. (a) Centers of plasmonic beams $\left(x_{0}, y_{0}\right)$ are steered with the velocity $v_{y}=1 \mathrm{~m} / \mathrm{s}$ and $E_{0}=0.2 \mathrm{MV} / \mathrm{cm}$. Centers of skyrmions $\left(q_{x, 1}, q_{y, 1}\right)$ and $\left(q_{x, 2}, q_{y, 2}\right)$ trapped by the plasmonic field follow two peaks of the plasmonic beam. (b) The critical velocity $v_{y}^{c}$ as a function of the plasmonic electric field $E_{0}$. The distance between peaks $l_{g}=37.5 \mathrm{~nm}$. (c) Deviation along $x$ axis of the positions of skyrmions centers $q_{x, 1(2)}^{0}$, from the centers of the plasmonic beam. For elevated electric field and stronger confinement $E_{0}$, the deviation is smaller. Here, $l_{g}=37.5 \mathrm{~nm}$ (blue dashed line). All results in this figure are from micromagnetic simulations.

The parameters of the Gaussian beam are $E_{0}=0.2 \mathrm{MV} / \mathrm{cm}$, $\sigma_{0}=25 \mathrm{~nm}$, and $l_{g}=37.5 \mathrm{~nm}$. We embed two skyrmions near the centers of two peaks $\left(x_{0} \pm l_{g}, y_{0}\right)=\left( \pm l_{g}, 0\right)$ [see Fig. 2(a)]. To test the robustness of the confinement, we evolve the system and track the positions of skyrmions via micromagnetic simulation. The relevant temperature profile, Fig. 2(b), has been extracted from the solution of the heat equation (see Supplemental Material [9]). One can expect that the effect of stochastic thermal noise is more pronounced for individual magnetic moments than for a skyrmion that is a "massive" object (i.e., topologically protected texture consisting of dozens of correlated magnetic moments). Indeed, comparing a single trajectory to the trajectory averaged over an ensemble of random realizations of the noise (see Supplemental Material [9]), we conclude that the impact of thermal fluctuations on the skyrmion trajectory is negligibly small for the parameters and temperatures considered in this Letter. Therefore, in a numerical simulation of skyrmion dynamics, we assumed $T=0$. Micromagnetic calculations have been also supported by the analytic model based on the Thiele equations for coupled skyrmions to be presented in the following. As follows from Fig. 2(a), the two skyrmions stay confined in the centers of the beam. Then we steer the center of the Gaussian beam $\left(x_{0}=0, y_{0}=v_{y} t\right)$ with the velocity $v_{y}=1 \mathrm{~m} / \mathrm{s}$. The drift of the plasmonic lattice drags both skyrmions [see Fig. 2(a)]. The corresponding dynamics of the skyrmions is plotted in Fig. 3(a). One can note a slight deviation of the interskyrmion distance along the $x$ axis from the distance between centers of the plasmonic lattice sites $\pm l_{g}$. This deviation is of the order of $q_{x, 2(1)}=$ $\pm 45 \mathrm{~nm}$ and can be ascribed to the repulsive interaction 
between two skyrmions. For a larger velocity of the beam center $v_{y}$, the oscillation of the skyrmion position around the peaks becomes stronger. At a critical oscillation amplitude, skyrmions overcome confinement and get released [see Fig. 2(c),(d)]. For $v_{y}=2.5 \mathrm{~m} / \mathrm{s}$, both skyrmions are still confined at the center of the plasmonic beam, and the confinement breaks down at the critical velocity $v_{y}^{c}=2.54 \mathrm{~m} / \mathrm{s}$. The critical velocity depends on the strength of confinement and is higher for the larger amplitude of the plasmonic electric field amplitude $E_{0}$, as shown in Fig. 3(b). The strong electric field $E_{0}$ suppresses the repulsive forces between skyrmions and keeps the skyrmions in the beam centers [see Fig. 3(c)]. It is known that skyrmions in the chiral ferromagnetic systems experience mutual repulsive interaction $[41,56,57]$. Thus, an important question pertains to the influence of the plasmonic lattice constant $l_{g}$ on the confinement effect. Since the repulsive force between the skyrmions decreases with increasing $l_{g}$, the critical velocity $v_{y}^{c}$ increases with $l_{g}$, as shown in Fig. 4(a). The corresponding distance between the skyrmions coincides with the distance between plasmonic lattice sites when $l_{g}$ is large enough [see Fig. 4(b)]. More precisely, when the plasmonic lattice parameter $l_{g}$ is much larger than the radius of the interaction between skyrmions $\xi$, the skyrmions are independent from each other. From the simulation results, we have extracted the interaction term in the free energy, $F_{M}(q)=F_{\text {tot }}-F_{1}-F_{2}$, where $F_{\text {tot }}$ is the energy of the entire magnetic texture and $F_{1,2}$ are the free energies of the isolated skyrmions [see Fig. 4(d)]. It turns out that the interaction energy between two skyrmions is well fitted by an exponential function $F_{M}(q)=A_{0} \exp (-q / \xi)$, and the decaying length is determined as $\xi=11 \mathrm{~nm}$ from our data. As we already mentioned, micromagnetic simulations were supported by the analytic model based on the Thiele equations for coupled skyrmions (see Supplemental Material [9]). The equilibrium state has been extracted from the static condition $\tau_{s, x(y)}=c F_{x(y), 1(2)}-B e_{x(y), 1(2)}=0$, where $F_{x(y), 1(2)}$ is the interaction force. Exploiting the inhomogeneous electric field in the simulation, we set up two skyrmions around: $q_{x, 1(2)}=-(+) q_{s}, q_{x, 1(2)}=+(-) q_{s}$. The dependence of $\tau_{s}$ on the plasmonic lattice constant is plotted in Fig. 4(c). For $E_{0}=0.8 \mathrm{MV} / \mathrm{cm}$ and $l_{g}=37.5 \mathrm{~nm}$, we find that the condition $\tau_{s, x}=0$ holds for $q_{s}=41 \mathrm{~nm}$, pointing out the balance between the IET and the interaction force. The model estimation of the equilibrium state precisely fits results of micromagnetic calculations in Fig. 4(b). Then, moving the laser electric field $y_{0}=v_{y} t$ with time, the Thiele equation can well describe the drag motion of two skyrmions for small $v_{y}$, as shown in Fig. 2(d). Increasing $v_{y}$ to a large enough value, the left skyrmion is pushed out of the laser range due to the repulsive effect.

In summary, we proposed a model of a plasmonic skyrmion lattice where the skyrmions are confined to
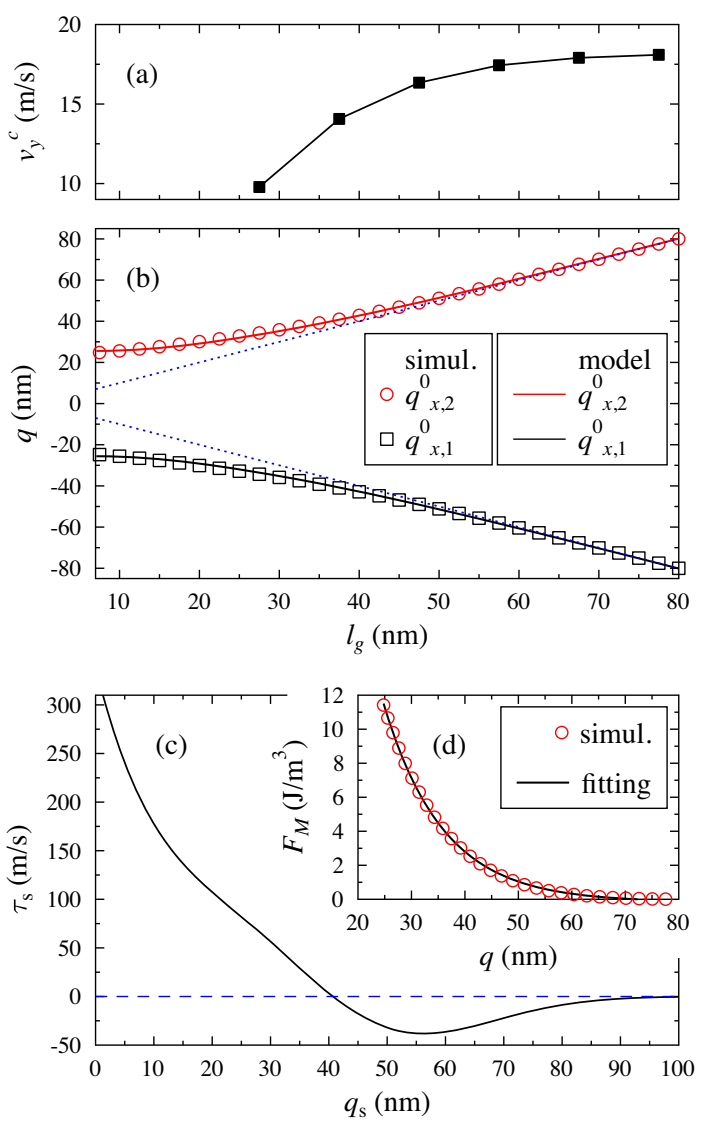

FIG. 4. (a) The critical velocity $v_{y}^{c}$ as a function of the plasmonic lattice constant $l_{g}$. The electric field $E_{0}=0.8 \mathrm{MV} / \mathrm{cm}$. (b) Positions of the skyrmion centers $q_{x, 1(2)}^{0}$ with increasing plasmonic lattice constant $l_{g}$. The blue dotted lines show the positions of the two plasmonic peaks. The amplitude of the plasmonic field $E_{0}=0.8 \mathrm{MV} / \mathrm{cm}$. (c) The total driving force $\tau_{s, x}$ in the Thiele equation as a function of skyrmion position $q_{s}$. (d) Dependence of the magnetic free energy density $F_{M}$ on the distance $2 q$ between two skyrmions, $2 q=q_{x, 1}^{0}-q_{x, 2}^{0}$. The magnetic free energy is extracted from the simulation results. The interaction part of the free energy $F_{M}(q)$ precisely fits to the exponential function $F_{M}=$ $A_{0} \exp (-q / \xi)$ with $A_{0}=110 \mathrm{~J} / \mathrm{m}^{3}$ and $\xi=11 \mathrm{~nm}$. The distance between skyrmion is controlled by varying distance between the plasmonic beams $l_{g}$ for (a) and (b).

plasmonic sites created by a four-laser beam. The confinement mechanism is based on the magnetoelectric coupling and also involves the plasmon electric field. Micromagnetic simulations have been supported by numerical solutions of the Thiele equations, which describe the motion of rigid skyrmions in the electric field. We found nice agreement between the results from simulations and those from the analytical approach, which indicates that skyrmion deformation is rather insignificant. The lattice of skyrmions trapped in plasmonic sites can be viewed as a new state of matter. In a certain sense, such a state is reminiscent of cold atom lattices. However, in the lattice sites, we have skyrmions instead of atoms. Moreover, this state can also be considered as a lattice of coupled skyrmion-plasmon states. 
An important issue is the dipolar coupling between skyrmions that leads to collective phenomena when the characteristic radius of the skyrmion-skyrmion interaction exceeds the distance between plasmonic lattice sites. Since the latter distance can be externally tuned, the plasmonic skyrmion lattices enable investigation as well as exploitation of these collective phenomena. Our system combines the unique magnetic properties of skyrmions with the ease and versatility of optical control. For example, the desired modification of the four-wave plasmonic interference pattern dynamically rearranges the skyrmionic lattice, while this is impossible in the trapping protocols discussed before. In particular, the collective magnonic modes of the trapped interacting skyrmions can be controlled through the geometry of the plasmonic lattice. The experimental study of the morphology of the skyrmion texture requires high finesse sensors, i.e., nitrogen-vacancy centers. Trapping of skyrmions in the plasmonic lattice may reduce the invasive effects of measurements (the drift of the magnetic texture) and enhance the accuracy of measurements. These and other related problems will be considered in the future. We also believe that our work will stimulate further experimental and theoretical investigations in the field of plasmonic skyrmionics.

This work was supported by the National Science Center in Poland as a research Project No. DEC-2017/27/B/ST3/ 02881, by the DFG through the SFB-TRR 227, Shota Rustaveli National Science Foundation of Georgia (SRNSFG) (Grant No. FR-19-4049), the National Natural Science Foundation of China No. 11704415, and the Natural Science Foundation of Hunan Province of China No. 2018JJ3629. A. E. acknowledges financial support from DFG through priority program SPP1666 (Topological Insulators), SFB-TRR227, and OeAD Grants No. HR 07/ 2018 and No. PL 03/2018. S. S. P P. acknowledges funding from the European Research Council (ERC) under the European Union's Horizon 2020 research and innovation programme (grant agreement SORBET No. 670166) and the Deutsche Forschungsgemeinschaft (DFG; German Research Foundation) Project No. 403505322 under SPP2137. Funding by the project W26000 Nanostrukturierte Materialien is gratefully acknowledged.

[1] L. Novotny and B. Hecht, Principles of Nano-Optics (Cambridge University Press, Cambridge, England, 2012).

[2] V. Klimov, Nanoplasmonics (CRC Press, Boca Raton, 2014).

[3] J. R. Krenn, H. Ditlbacher, G. Schider, A. Hohenau, A. Leitner, and F. R. Aussenegg, J. Microsc. 209, 167 (2003).

[4] J. Breault-Turcot and J.-F. Masson, Anal. Bioanal. Chem. 403, 1477 (2012).

[5] H. Ditlbacher, J. R. Krenn, G. Schider, A. Leitner, and F. R. Aussenegg, Appl. Phys. Lett. 81, 1762 (2002).
[6] L. Du, D. Y. Lei, G. Yuan, H. Fang, X. Zhang, Q. Wang, D. Tang, C. Min, S. A. Maier, and X. Yuan, Sci. Rep. 3, 3064 (2013).

[7] P. Dvorak, T. Neuman, L. Brinek, T. Samoril, R. Kalousek, P. Dub, P. Varga, and T. Sikola, Nano Lett. 13, 2558 (2013).

[8] T. J. Davis, D. Janoschka, P. Dreher, B. Frank, F.-J. M. zu Heringdorf, and H. Giessen, Science 368, eaba6415 (2020).

[9] See Supplemental Material, which includes Refs. [1,10-28], at http://link.aps.org/supplemental/10.1103/PhysRevLett 125.227201 for details.

[10] G. M. Zaslavsky, The Physics of Chaos in Hamiltonian Systems (World Scientific, Singapore, 2007).

[11] L. Chotorlishvili and A. Ugulava, Physica (Amsterdam) 239D, 103 (2010).

[12] A. Ugulava, L. Chotorlishvili, and K. Nickoladze, Phys. Rev. E 71, 056211 (2005).

[13] L. Chotorlishvili, P. Schwab, and J. Berakdar, J. Phys. Condens. Matter 22, 036002 (2009).

[14] A. E. Siegman, Lasers University Science Books (Mill Valley, CA, 1986) 37, 169.

[15] U. Levy, Y. Silberberg, and N. Davidson, Adv. Opt. Photonics 11, 828 (2019).

[16] R. Dorn, S. Quabis, and G. Leuchs, Phys. Rev. Lett. 91, 233901 (2003).

[17] K. S. Youngworth and T. G. Brown, Opt. Express 7, 77 (2000).

[18] D. Bäuerle, in Laser Processing and Chemistry (Springer, New York, 2011), pp. 13-38.

[19] D. Bäuerle, in Laser Processing and Chemistry (Springer, New York, 2011), pp. 457-475.

[20] A. Bernand-Mantel, C. B. Muratov, and T. M. Simon, Phys. Rev. B 101, 045416 (2020).

[21] F. C. Ummelen, T. Lichtenberg, H. J. Swagten, and B. Koopmans, Appl. Phys. Lett. 115, 102402 (2019).

[22] M. Castro, D. Mancilla-Almonacid, J. Valdivia, and S. Allende, J. Phys. Condens. Matter 32, 175801 (2020).

[23] R. Qin and Y. Wang, New J. Phys. 20, 063029 (2018).

[24] H. Vigo-Cotrina and A. Guimarães, J. Magn. Magn. Mater. 489, 165406 (2019).

[25] N. Vidal-Silva, A. Riveros, and J. Escrig, J. Magn. Magn. Mater. 443, 116 (2017).

[26] S. Castillo-Sepúlveda, R. Corona, A. Nuñez, and D. Altbir, J. Magn. Magn. Mater. 484, 451 (2019).

[27] N. Vidal-Silva, A. Riveros, F. Tejo, J. Escrig, and D. Altbir, Appl. Phys. Lett. 115, 082405 (2019).

[28] M. B. Hahn, J. Phys. Commun. 3, 075009 (2019).

[29] B. M. Piette, B. J. Schroers, and W. Zakrzewski, Z. Phys. C 65, 165 (1995).

[30] R. Rajaraman, in Solitons and Instantons: An Introduction to Solitons and Instantons in Quantum Field Theory (North-Holland, Amsterdam, 1982).

[31] T. H. R. Skyrme, in Selected Papers, With Commentary, of Tony Hilton Royle Skyrme (World Scientific, Singapore, 1994), pp. 195-206.

[32] A. Belavin and A. Polyakov, JETP Lett. 22, 245 (1975).

[33] B. Barton-Singer, C. Ross, and B. J. Schroers, Commun. Math. Phys. 375, 2259 (2020).

[34] B. J. Schroers, Phys. Lett. B 356, 291 (1995). 
[35] S. Seki, X. Yu, S. Ishiwata, and Y. Tokura, Science 336, 198 (2012).

[36] M. N. Wilson, A. B. Butenko, A. N. Bogdanov, and T. L. Monchesky, Phys. Rev. B 89, 094411 (2014).

[37] C. Schütte and M. Garst, Phys. Rev. B 90, 094423 (2014).

[38] J. S. White, K. Prša, P. Huang, A. A. Omrani, I. Živković, M. Bartkowiak, H. Berger, A. Magrez, J. L. Gavilano, G. Nagy et al., Phys. Rev. Lett. 113, 107203 (2014).

[39] A. Derras-Chouk, E. M. Chudnovsky, and D. A. Garanin, Phys. Rev. B 98, 024423 (2018).

[40] S. Haldar, S. von Malottki, S. Meyer, P. F. Bessarab, and S. Heinze, Phys. Rev. B 98, 060413(R) (2018).

[41] A. Leonov and M. Mostovoy, Nat. Commun. 6, 8275 (2015).

[42] C. Psaroudaki, S. Hoffman, J. Klinovaja, and D. Loss, Phys. Rev. X 7, 041045 (2017).

[43] K. A. van Hoogdalem, Y. Tserkovnyak, and D. Loss, Phys. Rev. B 87, 024402 (2013).

[44] S. Rohart, J. Miltat, and A. Thiaville, Phys. Rev. B 93, 214412 (2016).

[45] A. Samoilenka and Y. Shnir, Phys. Rev. D 95, 045002 (2017).

[46] R. A. Battye and M. Haberichter, Phys. Rev. D 88, 125016 (2013).
[47] P. Jennings and T. Winyard, J. High Energy Phys. 1 (2014) 122.

[48] S. Tsesses, E. Ostrovsky, K. Cohen, B. Gjonaj, N. Lindner, and G. Bartal, Science 361, 993 (2018).

[49] K. L. Tiwari, J. Lavoie, T. Pereg-Barnea, and W. A. Coish, Phys. Rev. B 100, 125414 (2019).

[50] R. Takagi, X. Z. Yu, J. S. White, K. Shibata, Y. Kaneko, G. Tatara, H. M. Rønnow, Y. Tokura, and S. Seki, Phys. Rev. Lett. 120, 037203 (2018).

[51] T. Liu and G. Vignale, Phys. Rev. Lett. 106, 247203 (2011).

[52] H. Wang, J. Chen, T. Liu, J. Zhang, K. Baumgaertl, C. Guo, Y. Li, C. Liu, P. Che, S. Tu et al., Phys. Rev. Lett. 124, 027203 (2020).

[53] M. Mostovoy, Phys. Rev. Lett. 96, 067601 (2006).

[54] V. Risinggård, I. Kulagina, and J. Linder, Sci. Rep. 6, 31800 (2016).

[55] X.-g. Wang, L. Chotorlishvili, G.-h. Guo, C.-L. Jia, and J. Berakdar, Phys. Rev. B 99, 064426 (2019).

[56] S.-Z. Lin, C. Reichhardt, C. D. Batista, and A. Saxena, Phys. Rev. B 87, 214419 (2013).

[57] Y. A. Kharkov, O. P. Sushkov, and M. Mostovoy, Phys. Rev. Lett. 119, 207201 (2017). 